\title{
Plasma vortex reactor for production of heat energy and hydrogen
}

\author{
Anatolii Klimov ${ }^{1,2}$ Svetlana Kurushina $^{2}$, Nonna Molevich $^{2 ; 3}$, Denis Porfiriev $^{2 ; 3}$ and Igor \\ Zaversinskii ${ }^{*}$, \\ ${ }^{1}$ Joint Institute for High Temperatures RAS, Moscow, 125412, Russia \\ ${ }^{2}$ Samara National Research University, Samara, 443086, Russia \\ ${ }^{3}$ Lebedev Physical Institute, Samara, 443011, Russia
}

\begin{abstract}
Numerical simulation of the turbulent three-dimensional swirling flow structure in plasma vortex reactor is conducted. Flow and set up parameters correspond to the experimental conditions. Flow velocity and thermal fields have been obtained. A qualitative agreement between the results of the calculations and the experimental data for pure argon has been shown.
\end{abstract}

\section{Introduction}

Recently, the problem of creating efficient compact energy sources, including alternative ones, has become increasingly noticeable [1,2]. One of the most promising technologies is the use of plasma vortex reactor (PVR), which can be both heat and hydrogen generator.

Intensive studies of a plasma chemical reactor started a few years ago [2-4]. Peculiar properties of gas-dynamics and heat fluxes in the presence of longitudinal electric discharge in the swirl flow were studied in [5,6]. The test gases used in this experimental set are the following: argon, argon + water vapor, argon + water vapor + nickel micro- and nanoparticles. The coefficient of performance was found. It was shown that COP depends on the parameters of the electric discharge and vortex flow itself. At the same time, the flow structure is determined by a number of factors: the shape and location of the discharge electrodes, swirl number, and the relative mass flow rates of the components.

A numerical simulation of the turbulent vortex flow structure in a model duct in the presence of a model heat source is carried out in [7]. The heat capacity of the swirler, electrodes and nozzle elements, as well as the thermal conductivity of the walls were neglected. It is shown that the recirculation zone forms in such system and its characteristics strongly depend on the heat source power and the shape of electrodes and their location. A qualitative agreement between the results of the calculations and the experimental data [8] has been obtained. The numerical modelling has demonstrated that it is possible to configure electrodes in such a way as to ensure energy transfer mainly downstream from the interelectrode zone. It may be accomplished by the combination of thin anode and pipe-like cathode through which areas of different pressures are connected.

\footnotetext{
* Corresponding author: ipzav63@mail.ru
} 


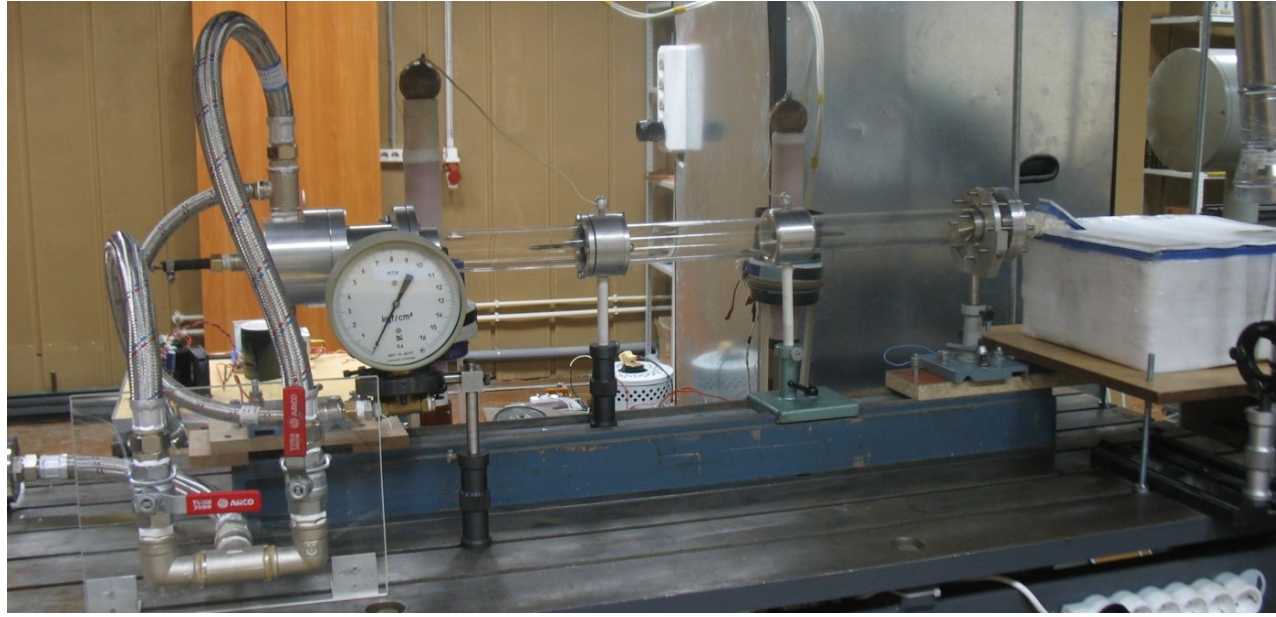

Fig.1. Experimental setup. General view.

Here, a simulation gasdynamic and thermal fields structure formed in a 3D unsteady viscous turbulent vortex flow of argon in the experimental setup [8] is carried out.

\section{Numerical modelling}

\subsection{Governing equations}

Standard unsteady Reynolds averaged Navier-Stokes (URANS) equations were used to describe the flow:

$$
\begin{gathered}
\frac{\partial \rho}{\partial t}+\frac{\partial\left(\rho v_{i}\right)}{\partial x_{i}}=0 \\
\frac{\partial\left(\rho v_{i}\right)}{\partial t}+\frac{\partial\left(\rho v_{i} v_{j}\right)}{\partial x_{i}}=-\frac{\partial P}{\partial x_{i}}+\frac{\partial}{\partial x_{i}}\left[\mu\left(\frac{\partial v_{j}}{\partial x_{i}}+\frac{\partial v_{i}}{\partial x_{j}}-\frac{2}{3} \delta_{i j} \frac{\partial v_{k}}{\partial x_{k}}\right)\right]+\frac{\partial}{\partial x_{i}}\left[-\rho \overline{v_{i}^{\prime} v_{j}^{\prime}}\right] \\
\frac{\partial(\rho E)}{\partial t}+\frac{\partial\left[v_{i}(\rho E+P)\right]}{\partial x_{i}}=\frac{\partial}{\partial x_{j}}\left[\left(\kappa+\frac{c_{P} \mu_{t}}{\operatorname{Pr}_{t}}\right) \frac{\partial T}{\partial x_{j}}+v_{i}\left(\tau_{i j}\right)_{e f f}\right]+N(\vec{x}), P=\frac{\rho T}{M},
\end{gathered}
$$

where $E=h-\frac{P}{\rho}+\frac{v^{2}}{2}$ is energy, $\left(\tau_{i j}\right)_{e f f}=\mu_{e f f}\left(\frac{\partial v_{j}}{\partial x_{i}}+\frac{\partial v_{i}}{\partial x_{j}}\right)-\frac{2}{3} \mu_{e f f} \frac{\partial v_{k}}{\partial x_{k}} \delta_{i j}$ is the deviatoric stress tensor; $\left\lfloor-\rho \overline{v_{i}^{\prime} v_{j}^{\prime}}\right\rfloor$ are the Reynolds stresses which must be modeled to close the set of equations, $v_{i}, v_{i}^{\prime}, \rho, T P, E$, and $h$ are the mean and fluctuating velocity components, density, temperature, pressure, total energy, and enthalpy, respectively; $N$ is the energy source, $\mu, \mu_{t}, \mu_{\text {eff }}$ are the molecular, turbulent, and effective viscosity coefficients, respectively; $c_{p}$ is the molar specific heat capacity at constant pressure; $\kappa$ is the thermal conductivity coefficient; and $P r_{t}$ is the turbulent Prandtl number.

The no-slip velocity and fixed temperature conditions were imposed along the tube and electrode surfaces. At the duct inlet and outlet, fixed mass flux conditions were used. Variation of the inlet turbulent intensity in the range of moderate values of $0-5 \%$ did not affect the flow structure discernibly. At the outlet, we imposed the boundary conditions with the static pressure equal to the atmosphere pressure.

Subsequently, we will solve the problem close to the experimental conditions [8], with ultimate goal of finding optimal configuration and location of electrodes. 


\subsection{Numerical simulation}

The numerical simulation of the non-stationary 3D turbulent swirling flow was made using the ANSYS FLUENT 15.0 program package, which solves system (1) using the finite volumes method. In our simulation, the Spalart-Allmaras model was used. A detailed analysis of the use of the turbulence models in similar problems is given in [8].

For spatial discretization of density, momentum, energy and turbulent quantities, a second-order upwind scheme is applied. The use of higher-order schemes has not changed the flow parameters considerably. The diffusion terms are central-differenced and secondorder accurate. We interpolate the pressure values at the faces using the PRESTO! scheme because of the strong swirl nature of the flow.

For transient terms, we used the fully implicit scheme of the second-order accuracy. Different pressure-velocity coupling schemes were tested and gave equal results. So, we used the SIMPLE scheme as the least resource intensive. The convergence was obtained when the residual reached $10^{-6}$ for the energy equation and $10^{-4}$ for the continuity equation, the momentum equation, and the equations for turbulent quantities.

The geometric modelling of the setup and the construction of the grid were carried out using the ANSYS Workbench package. The computational grid consisted of about $3 \cdot 10^{6}$ hexahedral cells, except for a small region, where the cells were tetrahedral, Fig 2.

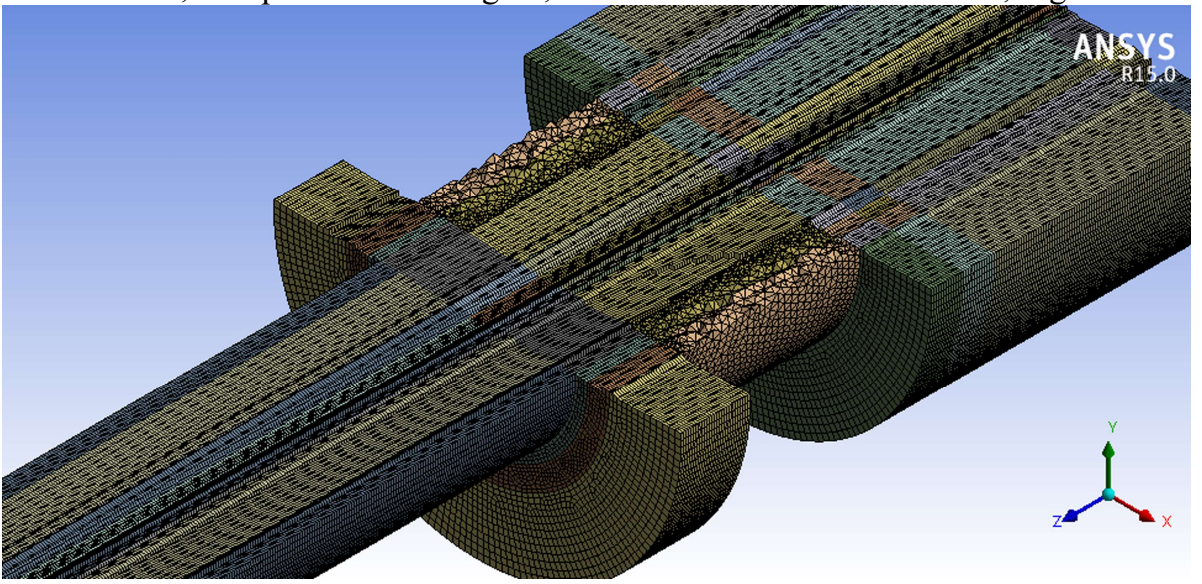

Fig. 2. The computational grid near the swirler.

The grid refinement did not considerably alter the gasdynamic parameters. Calculations with different values of time step from $1 \cdot 10^{-4} \mathrm{sec}$ to $1 \cdot 10^{-6} \mathrm{sec}$ were carried out. Finally, the time step was set equal to $5 \cdot 10^{-5} \mathrm{sec}$ since using lower values led only to an increase in the computational time.

The calculations have showed that the main features of the formation of the velocity and the thermal field are similar to founded in [7].

First, the structure of the flow is determined by the counterflow zone, typical for swirled flows [5,6], Fig. 3.

Secondly, using of two solid cylindrical electrodes leads to a significant drawback: between the electrodes, a dead zone is formed where the pressure and inertia forces almost prevent from the mass exchange with the outer flow. It leads to the interelectrode zone overheating and prevents for heat transfer downstream.

Thirdly, only using of thin anode and pipe-like cathode combination leads to optimal flow structure when the pressure gradient along the symmetry axis draws out the hot gas from the interelectrode area. The optimal electrodes location for this setup is close to the output of the PVR, Fig 4. 


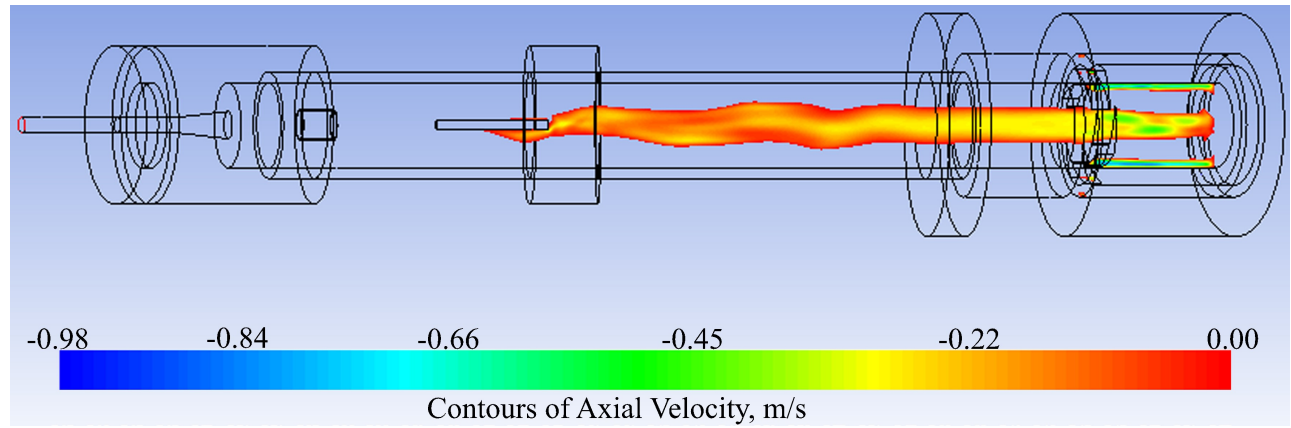

Fig. 3. Counterflow zone structure.

A hollow electrode is crucial for use in a PVP, since a solid one forms an obstacle that creates a negative axial pressure gradient and extends the counterflow zone. In this case, heat is effectively carried away downstream, so that the overheating zone isn't formed, the energy flux is directed mainly downstream from the cathode, Fig. 4.

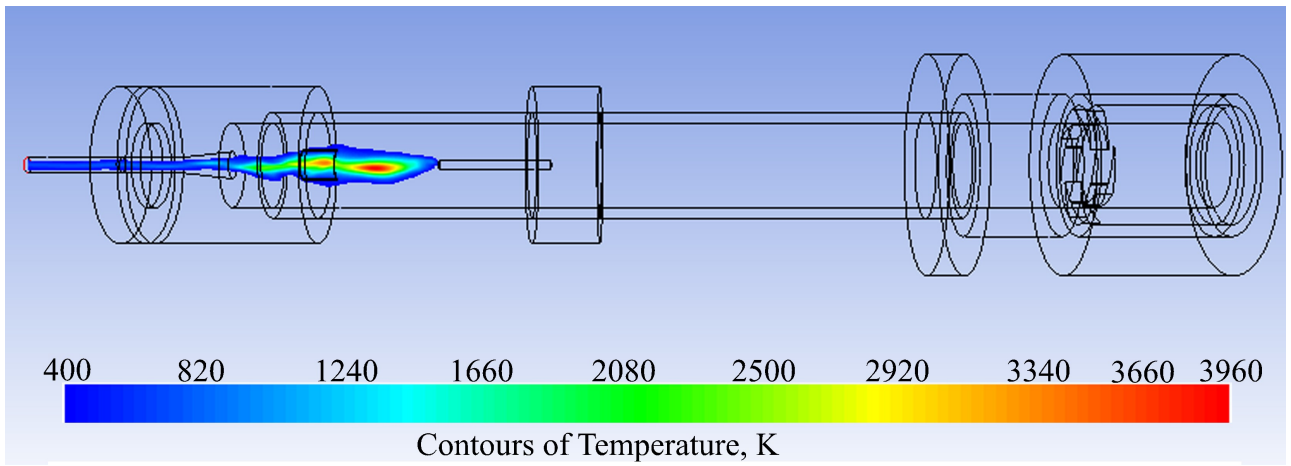

Fig. 4. Temperuture field at thin anode and hollow cathode located near the nozzle.

A qualitative agreement between the results of the calculations and the experimental data for pure argon [8] has been obtained. The next step in the simulation of the PVR is the calculation for real mixtures of $\mathrm{Ni}, \mathrm{H}_{2} \mathrm{O}$, Ar, taking into account the plasma-chemical kinetics of the discharge mixture components as well as thermal characteristics of the experimental set up.

The study was supported in part by the Ministry of Education and Science of Russia under the public contract with educational and research institutions within the projects 3.1158.2017/4.6, 14.Y26.31.0020 and by RFBR under grant 16-41-630591.

\section{References}

1. M. Jasiński, H. Dors, J. Nowakowska, J. Mizeraczyk, Chem. Listy, 102 (2008).

2. A. Klimov, V. Bitiurin, B. Tolkunov, V. Chinnov, S. Godin, A. Efimov, D. Kutuzov and L. Polyakov, AIAA Paper 2011-3285 (2011).

3. V.A. Bitiurin, A.I. Klimov, O.V. Korshunov, V.F. Chinnov, High Temperature, 52 (2014).

4. V.A. Bitiurin, A.I. Klimov, O.V. Korshunov, V.F. Chinnov, High Temperature, 53 (2015).

5. A. Gorbunova, A. Klimov, N. Molevich, I. Moralev, D. Porfiriev, S. Sugak, I. Zavershinskii, International J. Heat and Fluid Flow, 59 (2016).

6. $\quad$ N. Syred, Prog. Energy. Combust. Sci., 32 (2006). 
7. N.K. Belov, I.P. Zavershinskii, A.I. Klimov, N.E. Molevich, D.P. Porfiriev, B.N. Tolkunov. IOP Conf. Series: Journal of Physics: Conf. Series, 980 (2018).

8. P.N. Kazanskii, A.I. Klimov, N.E. Molevich, D.P. Porfiriev, I.P. Zavershinskii, IOP Conf. Series: Journal of Physics: Conf. Series 980 (2018). 